\section{Preprint}

not peer reviewed

\title{
An exploratory cluster \\ randomized controlled trial to \\ assess the impact of training \\ for teachers upon children's \\ motor competency outcomes
}

\begin{abstract}
Mann, S. ${ }^{1,2}$ Lucas, A. ${ }^{1}$, Wade, M. ${ }^{1,3}$, Shakespeare, J. ${ }^{1}$, Budzynski-Seymour, E. ${ }^{4}$, Conway, R. ${ }^{4}$, Steele, J. ${ }^{1,4}$ ${ }^{1}$ ukactive Research Institute, London, United Kingdom; ${ }^{2}$ Centre for Applied Biological and Exercise Sciences, Faculty of Health and Life Sciences, Coventry University, Coventry, United Kingdom; ${ }^{3}$ School of Sport Health and Applied Science, St Mary's University, Twickenham, United Kingdom; ${ }^{4}$ Faculty of Sport, Health, and Social Sciences, Solent University, Southampton, United Kingdom
\end{abstract}

Please cite as: Author, F., \& Author S. (YEAR). Title. SportRxiv. https://doi.org/XX.XXXX/osf.io/XXXXX
All authors have read and approved this version of the manuscript. This article was last modified on Month XX, YEAR.
Authors X@xtwitter and Y @ytwitter can be reached on Twitter. 


\section{INTRODUCTION}

Physical literacy has been defined by Whitehead (2019) as the "...motivation, confidence, physical competence, knowledge and understanding to value and take responsibility for engaging in physical activities for life". Physical literacy has often been considered a holistic concept across varying domains (Longmuir et al., 2015; Whitehead, 2019; Keegan et al., 2019); indeed, a recent Delphi study highlighted physical, psychological, cognitive, and social domains as key (Keegan et al., 2019), and in her most recent work discusses the contribution of affective, physical, and cognitive domains to the monist whole of physical literacy.

Though not without criticism (Almond, 2014), development of the physical domain is often a focus of physical education including, but not limited to, motor competence (Robinson et al., 2015). Despite the philosophical critiques, development of these are positively associated to physical activity participation (Morgan et al., 2013; Francis et al., 2016), fitness levels (Lubans et al., 2010), and maintenance of physical activity over time in children (Barnett et al., 2009; Lopes et al., 2011), in addition to increased physical activity participation in obese children (Morgan et al., 2008). Additionally, motor competence abilities have been associated to the sustained health of children with previous research demonstrating that children aged 6-14 who had greater proficiency in motor competence were more likely to have lower BMI and lower levels of obesity than those with poorer motor competence (Chalk et al., 2016; Lopes et al., 2014; Lopes et al., 2012).

School remains a valuable environment in which children can engage in physical activity and develop motor competence via their peers and teachers (Ryan and Deci, 2007). Indeed, the 
implementation of planned motor competency programs in schools has been shown to positively impact children's fundamental movement skills (Logan et al., 2012; Morgan et al., 2013). Thus, it has been argued that physical education (PE) lessons in primary schools should integrate physical domain elements of physical literacy (e.g. body control, locomotion, and manipulation skills) as they underpin development of motor competency (Higgs, 2010).

Despite the wider growth in popularity of physical literacy in schools, and more specifically motor competency, due to more traditional views of undertaking PE not all schools or school teachers are aware of how to integrate this into a PE lesson (Lundvall, 2015). Indeed, despite the success of specifically delivered motor competency related programmes (Logan et al., 2012; Morgan et al., 2013), there is less clarity on the impact of teaching training related interventions (Lander et al., 2017). This study aimed to address this issue, by working with targeted primary schools. This research focused on the physical domain in terms of motor competency. Specifically, this study aims to explore the effectiveness of a training programme for teachers in improving the motor competency aspects of children's physical literacy in primary schools in Buckinghamshire, by providing teachers with skills and resources to deliver motor competency outcomes efficiently.

\section{METHOD}

\section{Study Design}

A cluster randomised controlled trial was performed with schools randomised to either the intervention, or a wait-list control arm. The intervention was delivered at the level of the teachers 
within the schools, though outcomes were collected at the level of the children. As such, the trial represents the exploration of a 'train the teacher' intervention upon children's motor competency.

\section{Participants}

Participants were $(n=136)$ children aged between 5-7 years from eight primary schools across nine classes.

\section{Delivery}

Nine teachers, from randomly assigned schools, received specific training and resources during curriculum time. The training and resources provided were written by a specialist in early years and primary physical development. Furthermore, the sessions were constructed to align with the aims of the Early Years Foundation Stage and the National Curriculum of PE. The resources were specifically developed for this study and included lesson plans for a 30-week period (based on one lesson per week). The training and testing resources had previously been tested with 55 nurseries, pre-schools, and primary schools across the county over the past two years. The training took place in five settings over the academic year. Both the resources for 30 lessons, and the 3hour training workshop were intended to provide the teachers with the necessary skills to deliver age and stage appropriate opportunities that will allow schools to maximise the motor competency development of their pupils, resulting in them achieving outstanding PE lessons. 
Three of the teachers received the intervention training initially. As such, these teachers were provided with training to deliver such PE lessons as described above to their classes. Six of the teachers formed a wait-list control group (who would receive the intervention the next year) whose classes provided a comparison group. This is critical given the fact that children's fundamental movement skills develop with maturation (and age; Whitehead 2001) therefore to determine the success of the intervention children's motor competency scores must be compared to those maturing at the same rate.

\section{Ethics and Consent}

Informed consent was obtained from schools and teachers, and subsequently from parents/guardians of all potential participants. Further, children provided informed assent to participate in the testing, though participation in PE sessions was considered part of normal school educational delivery. This is a model that was been developed through consultation with Public Health England and the Office for Standards in Education, Children's Services and Skills (Ofsted) to ensure complete anonymity.

All data were anonymised immediately following collection and stored on secure systems approved by Ofsted information governance team. Opt-out forms and any paper files were stored in locked cabinets within the schools, and electronic files stored on password protected computers, in both cases in accordance with the Ofsted information governance team. All hard 
copies of files were destroyed upon completion of the investigation. Institutional ethical approval was granted for this study.

\section{Measures}

Physical literacy, focusing specifically on motor competency, was the measure employed to assess the impact of the teacher training programme. Measures of motor competency were taken from the Canadian 'Physical Literacy Assessment for Youth' methodology (Canadian Sport for Life, 2013). Children were scored in accordance to their proficiency, whereby they were grouped into categories of being emerging, competent or proficient in a particular skill. External coaches who were blinded to the schools grouping (e.g. as control or intervention) completed the scoring, with reference to a standardised guide which allowed scoring on a one-ten scale. This scale was adapted from the original methodology to allow specificity in assessment and to increase the sensitivity of the measure to change over time. This assessment has been found to have excellent inter-rater objectivity $(\mathrm{ICC}=0.99)$ for skill score and substantial for completion time (ICC=0.69), with reliability over 'long intervals' (8-14 days) found to be substantial (ICC=0.74) for skill score and excellent for completion time (ICC=0.82; Longmuir et al., 2017). All testing was conducted at same time of the year for both groups.

Locomotion: This task required children to run five metres to a cone, stop, turn around, and run back. The task was then repeated with children being required to run backwards to the start point. 
Coaches scored children in relation to a guide noting the ease and synchronization of movement (e.g. stumbling, synchronized arm and leg movement), the sprint speed, the acceleration and ability to pivot and change direction (e.g. to turn around).

Throwing : This task required children to throw a tennis ball against a wall. The aim was to hit the wall above a marker indicating 1.5 metres from the ground, and to throw it hard enough so that it bounced back over their heads. Children had to remain behind a line marked three metres from the wall. The second task was adapted for ease, requiring children to throw the tennis ball against the wall above a marker of 1.5 metres and catch it, standing a distance of 3 metres from the wall. Coaches scored children in relation to a guide noting the distance, trajectory and speed of the ball (e.g. did it successful hit the wall and return), the rotation of the trunk, the ease of movement (e.g. disjointed or smooth limb motion), and the catching ability.

Kicking: This task required children to kick a football (size 4) over a marker indicating one metre from the ground, from an area four metres from the wall. The task was then repeated, but instead required children to drop kick the ball, over the one metre marker from a distance of four metres. Coaches scored children in relation to a guide noting the distance, trajectory, speed and elevation of the ball, the synchronization between upper and lower body movement, foot contact and control with the ball.

Balancing: This task required children to hop from one foot to the other and back onto the original leg, keeping in line with two cones placed one metre apart, before balancing on one foot when stopping (kinetic balance). The second task required children to stand on one foot and stretch donating at www.storkinesiology.org/annual 
their arm up in the air and point their finger (static balance). Next, they had to bend down and touch the ground in front of their toes while keeping balanced, before standing back up and extending their arm to the air. This was followed by the same movement, but touching the ground behind their heel. Coaches scored children in relation to a guide noting ability to balance, loss of balance (e.g. touching the ground), control and fluidity of movement, foot placement, and strain (e.g. wobbling) of upper and lower limbs.

\section{Statistical Analysis}

All analysis was performed using $\mathrm{R}$ (version 3.5.0; $\mathrm{R}$ Core Development Team, https://www.r-project.org/). Linear mixed models were used for analysis. 'Group' was modelled as a fixed factor with pre-scores included as a fixed covariate. Random intercepts by class were included. Due to the inclusion of both fixed and random effects Restricted Maximum Likelihood estimation was used. The main effects of 'group' were examined upon absolute changes (postminus pre-scores i.e. $\Delta$ ) in the outcome measures for motor competency as both the sum of scores, and for individual components. Analysis was considered exploratory with statistical significance accepted at $\alpha=0.05$ yet any effects treated with appropriate caution and skepticism. Estimated marginal means and $95 \%$ confidence intervals were calculated for $\Delta$ from the models for each group and presented for consideration of the point estimates of effects along with their precision. Data and code are available: https://osf.io/bz9vn/. 


\section{Results}

Pre-post intervention means for the control and intervention groups indicate an increase in motor competency across all participants (Table 1). The means indicate that scores from intervention schools increased to a greater extent over the academic year than those of control. However, results from the multilevel analysis suggested that most changes in outcomes did not statistically significantly differ between groups when accounting for both between school fixed effects and pre-score values as covariates (Table 2). Figure 1 presents the estimated marginal means from the models for each outcome and group which suggests that for a number of the outcomes both control and intervention groups improved, though there seems to be a tendency for the effect estimate and its precision to include potentially higher values for change scores in the intervention group compared to the control group across most variables. However, there was some overlap in likely population estimates between groups for all outcomes.

Table 1. Physical Literacy score by measure

\begin{tabular}{lcccccccc}
\hline & \multicolumn{3}{c}{ Pre } & \multicolumn{2}{c}{ Control } & \multicolumn{2}{c}{ Post } & \multicolumn{3}{c}{ Pre } & Post \\
& Mean & SD & Mean & SD & Mean & SD & Mean & SD \\
\hline Running Forward & 6.25 & 1.02 & 6.89 & 1.17 & 5.88 & 1.22 & 7.12 & 0.83 \\
Running Backwards & 6.11 & 1.07 & 6.87 & 1.13 & 5.93 & 1.08 & 6.59 & 1.02 \\
Throwing & 6.58 & 1.25 & 7.08 & 1.46 & 6.25 & 1.27 & 7.41 & 0.98 \\
Throwing and Catching & 5.56 & 1.36 & 6.53 & 1.49 & 5.46 & 1.37 & 6.92 & 1.48 \\
Kicking Ground & 6.56 & 1.45 & 6.46 & 1.57 & 6.05 & 1.12 & 7.71 & 1.12 \\
Kicking Drop & 4.97 & 1.79 & 6.07 & 1.54 & 4.96 & 1.57 & 6.58 & 1.50 \\
Balance Static & 5.75 & 1.27 & 6.49 & 1.46 & 5.73 & 1.07 & 6.96 & 1.22 \\
Balance Kinetic & 5.99 & 1.33 & 6.37 & 1.38 & 5.63 & 1.32 & 6.96 & 1.40 \\
Average & $\mathbf{5 . 9 7}$ & $\mathbf{0 . 6 6}$ & $\mathbf{6 . 5 9}$ & $\mathbf{0 . 9 1}$ & $\mathbf{7 . 1 2}$ & $\mathbf{0 . 8 3}$ & $\mathbf{7 . 0 0}$ & $\mathbf{0 . 8 6}$ \\
Sum Scores & $\mathbf{4 2 . 0 2}$ & $\mathbf{4 . 7 7}$ & $\mathbf{4 6 . 4 0}$ & $\mathbf{6 . 6 7}$ & $\mathbf{4 0 . 1 7}$ & $\mathbf{4 . 2 2}$ & $\mathbf{4 9 . 4 5}$ & $\mathbf{5 . 8 9}$ \\
\hline
\end{tabular}


Table 2. Mixed model results.

\begin{tabular}{lcccc}
\hline & $\begin{array}{c}\text { Mean difference } \\
(\text { Int } \Delta-\text { Cont } \Delta)\end{array}$ & $95 \% C l s$ & F & \\
& 0.330 & -0.692 to 1.351 & 0.589 & 0.468 \\
\hline Running Forward & -0.241 & -1.090 to -0.609 & 0.488 & 0.512 \\
Running Backwards & 0.331 & -0.839 to 1.502 & 0.448 & 0.523 \\
Throwing & 0.446 & -0.361 to 1.252 & 1.681 & 0.234 \\
Throwing and Catching & 1.474 & 0.419 to 2.529 & 11.134 & 0.013 \\
Kicking Ground & 0.553 & -0.398 to -1.503 & 1.917 & 0.210 \\
Kicking Drop & 0.437 & -0.557 to 1.450 & 1.130 & 0.330 \\
Balance Static & 0.590 & -0.486 to 1.666 & 1.720 & 0.233 \\
Balance Kinetic & 0.542 & -0.276 to 1.360 & 1.792 & 0.226 \\
Average & -5.303 & -14.852 to 4.246 & 2.473 & 0.161 \\
Sum & & &
\end{tabular}
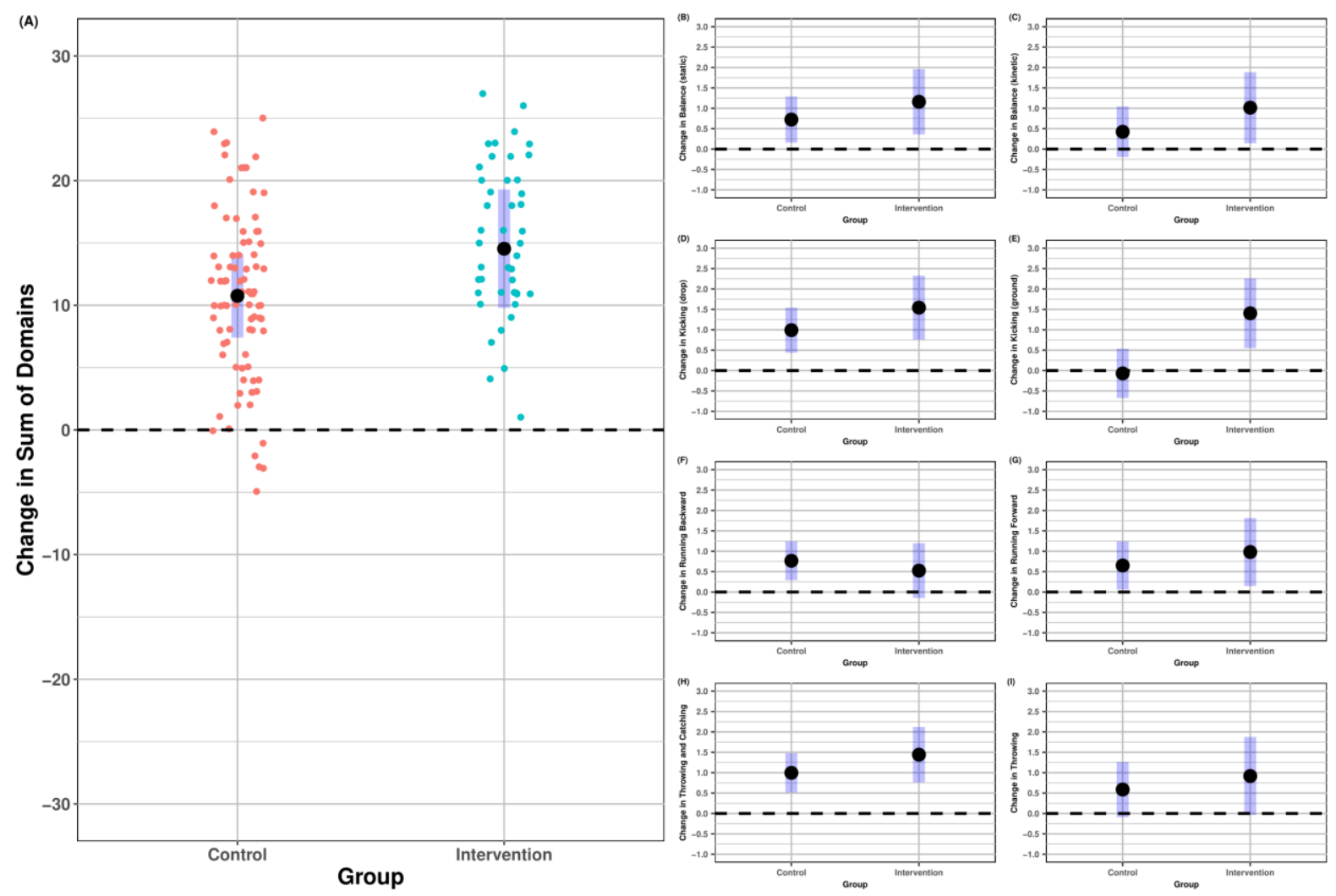

- Control - Intervention

Figure 1. Intervention vs control estmated marginal means for change $(\Delta)$ for each outcome. 


\section{Discussion}

The primary purpose of this investigation was to examine whether children's motor competency would benefit from a programme targeted at providing primary school teachers more skills and knowledge in delivering physical literacy related outcomes and activities. Overall, there was an increase in motor competency scores across all participants. Intervention schools saw a slightly greater increase in 7 of 8 scores in comparison to their control counterparts though this did not significantly differ. This implies that children's involvement in their PE classes throughout the academic year and their natural maturation helps to improve their longer-term motor competency. However, while this improves motor competency with time, a programme specifically upskilling teachers to provide motor competency related PE sessions may offer some small benefit.

It is suggested that motor development contributes as a key aspect in the total development and well-being of children and must be addressed in the early years of childhood (Pienaar, 2009). However, whilst there is evidence to suggest that motor skills are partially developed naturally as a child ages and matures, recent research postulates a more complex interaction between the movement task, biology of the child, and the environment (Malina et al., 2004; Stodden et al., 2008; Livonen and Sääkslahti, 2013). These three subsystems individually and mutually either encourage or discourage skill acquisition (Gallahue and Ozmun, 2012). As a result, young children have various levels of motor competence, primarily because of differences in experience (Stodden et al., 2008). 
Considering that children have the developmental potential to master most of the fundamental movement skills by the age of six years, it a cause for concern that children aged between 5-7 within this study had not previously attained sufficient motor competence. If children are not proficient in basic motor skills, then they may have limited opportunities for engagement in physical activities in later life, thus highlighting the importance of structured training for the acquisition of these skills (Stodden et al., 2008; Stodden et al., 2014).

The school setting would seem to be the most opportune location for structured training due to being able to provide greater access to resources (i.e. equipment, space and education) than that of the home setting (Kirk and Rhodes, 2011). Providing a developmentally appropriate environment that offers encouragement and opportunities for skill-specific practice may aid in the progress toward mature forms of motor competence (Livonen and Sääkslahti, 2013). Therefore, providing teachers with skills and knowledge in delivering motor competency related outcomes and activities help to increase children's motor competency. Yet, the results of this study suggest that at best this may only exert a small effect upon children's motor competency. Considering prior evidence suggesting that school based structured motor competency interventions are effective (Logan et al., 2012; Morgan et al., 2013), this may be due the implementation here of a 'train the teacher' intervention for which evidence is less clear regarding its effectiveness (Lander et al., 2017). Unfortunately, we did not conduct an evaluation of the fidelity of teachers implementation of movement competency related PE sessions and as such these may not have been implemented as intended. However, recent work has shown that, once 
upskilled, teachers continue to implement delivery aimed at improving motor competency supporting sustainability (Lander et al., 2020) and possibly that over time greater effects might manifest on children's motor competency.

Though upskilling general primary school teachers produced some small improvements in motor competency, it is possible that a structured programme delivered by specifically trained external deliverers would have elicited greater improvements in these scores, further reducing the developmental delay in motor competency within this population. However, the initial upskilling of primary school teachers clearly results in less resources (i.e. time, money and external staff) required over the long-term, thus providing a suitable alternative to the use of external resources. Previous literature has demonstrated that providing education and material regarding motor competence to school staff resulted in improvements in children's motor competency (Hardy et al., 2010). The programme did not include any compulsory structured lessons, demonstrating that regardless of the programme details, educating staff about the individual components of motor competence can have a positive effect. Though our results only suggest a small effect, when weighed against the resource implications of more intensive interventions delivered by trained and qualified professionals this may be deemed worthwhile. Future research should certainly look to test this small effect in larger samples in addition to considering the comparative cost-effectiveness against more intensive interventions.

Enhancing motor competency is fundamental in influencing one's engagement in physical activities (Ryan and Deci, 2007). Research indicates that the more physically literate a child is the 
more likely they will stay physically active, pertain to long-term health and wellbeing, and develop essential motor co-ordination skills (Morgan et al., 2013). Fundamental movement skills are typically composed of locomotor (e.g. running and hopping), object control (e.g. throwing, catching and kicking) and stability (e.g. balancing; Lubans et al. 2010), each of which were measured in this study. Analysis of specific motor competency components in the present study indicated that children were most proficient in balancing and object control skills, in particular kicking, and least proficient in locomotion. Not only were the children most competent within these skills initially, they also showed the greatest improvement in these skills within the intervention programme. This indicates that locomotor skills may be harder to master due to being greatly influenced by underlying capabilities (Westendorp et al., 2011; Burton and Rodgerson, 2001). In contrast, easier tasks, such as kicking, throwing and static balancing appear to reveal primarily time-related effects caused by familiarisation potentially explaining the greater elicited changes in these proficiencies (Donath et al., 2015).

It is postulated that proficiency in object control skills in childhood may be influential in building positive perception of sports competence, in turn increasing adolescent physical activity engagement (Barnett et al, 2008). On the other hand, it has been shown that there is limited evidence for locomotor proficiency in childhood and adolescence, indicating that gaining object control skills are potentially more important than locomotor skills as they track through to adolescence (Zask et al., 2012). This could be attributed to the fact that locomotor ability is more variable than object control ability due to the influences of underlying capabilities (Burton and donating at www.storkinesiology.org/annual 
Rodgerson, 2001). However, it is recommended that locomotor skills are not disregarded but that they are integrated alongside object control skills in learning activities (Zask et al., 2012). Considering this, a specific focus should remain on promoting these skills in future interventions to promote sustained physical activity levels and a consistently active lifestyle in children. Finally, in order to ensure physical literacy is understood in its complete capacity, any intervention could look to incorporate measures of across wider domains, in addition to motor competency. This would not only provide a more in-depth picture of physical literacy as per its definition, it would also allow one to explore how these elements correlate and possibly enhance the small effects seen here (Longmuir et al., 2015; Whitehead, 2001).

The present studies limitations need to be considered. Firstly, as noted there was no measure of fidelity as there was a lack of monitoring around this area. It is unknown therefore how many hours of training for teachers, or the degree to which they implement what they have been taught, result in motor competency improvement. Furthermore, the measures were not taken again at a later date, meaning that it is unclear to what the more long-term results may have been, and as monitoring ended for financial and logistic reasons after the initial intervention period meaning we were unable to include the wait-list control intervention period to increase power. It would also have been useful to record both the previous experiences of the staff involved and to monitor the extracurricular activities of the children to consider the impact of other sporting sessions on their motor competency skill development. 


\section{Conclusion}

The present exploratory study suggests that children's motor competency scores improve through both regular PE or PE delivered by staff specifically trained in motor competency related outcomes and activities. However, though not statistically significantly different, outcomes tended to favour the intervention suggesting children may experience a small benefit from participation in a motor skill intervention delivered by trained teachers. These findings may have implications for informing future programmes surrounding physical literacy. Upskilling of staff with the knowledge and resources necessary to enhance children's physical activity at school may at best have a small impact, though is generally easy and uncostly to implement. Further research with sufficient power should test this small effect. Also, development of this programme, additionally focusing on the elements of motivation and confidence, may be warranted in order to better understand and promote physical literacy.

\section{Contributions}

Contributed to conception and design: SM, MW, JSh, AL

Contributed to acquisition of data: SM, MW, AL

Contributed to analysis and interpretation of data: SW, MW, JSt, EBS

Drafted and/or revised the article: SW, MW, EBS, RC, JSt

Approved the submitted version for publication: $S<, A L, M W$, JSh, EBS, RC, JSt

\section{Acknowledgements}

The authors wish to thank Tom Burton and Sarah Preston at Buckinghamshire County Council, in addition to Chris Gregory from Leap (Bucks and MK Sport and Activity Partnership), for facilitating this research. Further thanks are extended to all the participants and participating schools. 


\title{
Funding information
}

This work was supported by Buckinghamshire County Council and Leap (Bucks and MK Sport and Activity Partnership).

\section{Data and Supplementary Material Accessibility}

\author{
https://osf.io/bz9vn/
}

\section{REFERENCES}

1. Almond, L. (2014). Serious flaws in an FMS interpretation of physical literacy. Science and Sports, 29, S60

2. Barnett, L. M., Morgan, P. J., van Beurden, E., \& Beard, J. R. (2008). Perceived sports competence mediates the relationship between childhood motor skill proficiency and adolescent physical activity and fitness: a longitudinal assessment. The International Journal of Behavioral Nutrition and Physical Activity, 5, 40

3. Barnett, L. M., van Beurden, E., Morgan, P. J., Brooks, L. O., \& Beard, J. R. (2009). Childhood motor skill proficiency as a predictor of adolescent physical activity. The Journal of Adolescent Health, 44(3), 252-259

4. Burton, A.W., \& Rodgerson, R.W. (2001). New Perspectives on the Assessment of Movement Skills and Motor Abilities. Adapted Physical Activity Quarterly, 18, 347365

5. Canadian Sport for Life. (2013). Physical Literacy Basic Physical Literacy Assessment for Youth (PLAY). Victoria, BC.

6. Case, A., Fertig, A., \& Paxson, C. (2005). The lasting impact of childhood health and circumstance. Journal of Health Economics, 24(2), 365-389

7. Chak, A., Fu, L., Cobley, S.P., \& Sanders, R.H. (2016). Motor Coordination Training and Pedagogical Approach for Combating Childhood Obesity. Open Journal of Social Sciences, 4, 1-12

8. Donath, L., Faude, O., Hagmann, S., Roth, R., \& Zahner, L. (2015). Fundamental movement skills in preschoolers: a randomized controlled trial targeting object control proficiency. Child: Care, Health and Development, 41(6), 1179-1187

9. Francis, C. E., Longmuir, P. E., Boyer, C., Andersen, L. B., Barnes, J. D., Boiarskaia, E., Cairney, J., Faigenbaum, A. D., Faulkner, G., Hands, B. P., Hay, J. A., Janssen, I., Katzmarzyk, P. T., Kemper, H. C., Knudson, D., Lloyd, M., McKenzie, T. L., Olds, T. S., Sacheck, J. M., Shephard, R. J., ... Tremblay, M. S. (2016). The Canadian Assessment of Physical Literacy: Development of a Model of Children's Capacity for a Healthy, 
Active Lifestyle Through a Delphi Process. Journal of Physical Activity \& Health, 13(2), 214-222

10. Gallahue, D.L, \& Donnelly, F.C. (2003). Developmental Physical Education for All Children. Champaign, IL: Human Kinetics.

11. Gallahue, D.L, \& Ozmun, J.C. (2012). Understanding Motor Development: Infants, Children, Adolescents, Adults. $7^{\text {th }}$ ed. Boston, MA: McGraw-Hill.

12. Goodway, J., Brian, A., Chang, S.H., Famelia, R., Tsuda, E., \& Robinson, L. (2013). Promoting Physical Literacy in the Early Years Through Project SKIP. ICSSPE Bulletin, $65,122-30$

13. Hardy, L. L., King, L., Kelly, B., Farrell, L., \& Howlett, S. (2010). Munch and Move: evaluation of a preschool healthy eating and movement skill program. The International Journal of Behavioral Nutrition and Physical Activity, 7, 80.

14. Higgs, C. (2010). Physical Literacy -- Two Approaches, One Concept. Phys \& Health Ed J, 76, 6

15. Keegan, R. J., Barnett, L. M., Dudley, D. A., Telford, R. D., Lubans, D. R., Bryant, A. S., Roberts, W. M., Morgan, P. J., Schranz, N. K., Weissensteiner, J. R., Vella, S. A., Salmon, J., Ziviani, J., Okely, A. D., Wainwright, N. \& Evans, J. R. (2019). Defining physical literacy for application in Australia: A modified delphi method. Journal of Teaching in Physical Education, 38 (2), 105-118.

16. Kirk, M. A., \& Rhodes, R. E. (2011). Motor skill interventions to improve fundamental movement skills of preschoolers with developmental delay. Adapted Physical Activity Quarterly, 28(3), 210-232

17. Lander N, Eather N, Morgan PJ, Salmon J, Barnett LM. Characteristics of Teacher Training in School-Based Physical Education Interventions to Improve Fundamental Movement Skills and/or Physical Activity: A Systematic Review. Sports Med. 2017 Jan;47(1):135-161. doi: 10.1007/s40279-016-0561-6. PMID: 27294354.

18. Lander, N., Salmon, J., Morgan, P. J., Symington, N., \& Barnett, L. M. (2020). Threeyear maintenance of a teacher-led programme targeting motor competency in early adolescent girls. Journal of Sport Sciences, Epub ahead of print.

19. Livonen, S., \& Sääkslahti, A.K. (2013). Preschool children's fundamental motor skills: a review of significant determinants. Early Child Development and Care, 184, 11071126

20. Logan, S. W., Robinson, L. E., Wilson, A. E., \& Lucas, W. A. (2012). Getting the fundamentals of movement: a meta-analysis of the effectiveness of motor skill interventions in children. Child: Care, Health and Development, 38(3), 305-315. 
21. Longmuir, P.E., Boyer, C., Lloyd, M., Yang, Y., Boiarskaia, E., Zhu, W., \& Tremblay, M.S. (2015). The Canadian Assessment of Physical Literacy: Methods for Children in Grades 4 to 6 (8 to 12 Years). BMC Public Health, 15, 767

22. Longmuir, P. E., Boyer, C., Lloyd, M., Borghese, M. M., Knight, E., Saunders, T. J., Boiarskaia, E., Zhu, W., \& Tremblay, M. S. (2017). Canadian Agility and Movement Skill Assessment (CAMSA): Validity, objectivity, and reliability evidence for children 8-12 years of age. Journal of sport and health science, 6(2), 231-240. https://doi.org/10.1016/j.jshs.2015.11.004

23. Lopes, V. P., Rodrigues, L. P., Maia, J. A., \& Malina, R. M. (2011). Motor coordination as predictor of physical activity in childhood. Scandinavian Journal of Medicine \& Science in Sports, 21(5), 663-669

24. Lopes, V. P., Stodden, D. F., \& Rodrigues, L. P. (2014). Weight status is associated with cross-sectional trajectories of motor co-ordination across childhood. Child: Care, Health and Development, 40(6), 891-899

25. Lubans, D. R., Morgan, P. J., Cliff, D. P., Barnett, L. M., \& Okely, A. D. (2010). Fundamental movement skills in children and adolescents: review of associated health benefits. Sports Medicine, 40(12), 1019-1035

26. Lundvall, S. (2015). Physical Literacy in the Field of Physical Education - A Challenge and a Possibility. Journal of Sport and Health Science, 4, 113-118

27. Malina, R.M., Bouchard, C., \& Bar-Or, O. (2004). Growth, Maturation and Physical Activity. $2^{\text {nd }}$ ed. Champaign, IL: Human Kinetics.

28. Morgan PJ, Okely AD, Cliff DP, Jones RA, Baur LA. Correlates of objectively measured physical activity in obese children. Obesity (Silver Spring). 2008;16(12):26342641

29. Morgan, P.J., Barnett, L.M., Cliff, D.P., Okely, A.D., Scott, H.A, Cohen, K.E., \& Lubans, D.R. (2013). Fundamental Movement Skill Interventions in Youth: A Systematic Review and Meta-Analysis. Pediatrics, 132, e1361-e1383

30. Pienaar, A. (2009). Kinderkinetics: An investment in the total well-being of children. South African Journal for Research in Sport, Physical Education and Recreation, 31, 49-67

31. Robinson, L. E., Stodden, D. F., Barnett, L. M., Lopes, V. P., Logan, S. W., Rodrigues, L. P., \& D'Hondt, E. (2015). Motor Competence and its Effect on Positive Developmental Trajectories of Health. Sports medicine (Auckland, N.Z.), 45(9), 1273-1284.

32. Ryan, R.M., \& Deci, E.L. (2007). Active Human Nature: Self-Determination Theory and the Promotion and Maintenance of Sport, Exercise, and Health. In M. S. Hagger, 
\& N. L. D. Chatzisarantis (Eds.), Intrinsic Motivation and Self-Determination in Exercise and Sport (pp. 1-19). Leeds: Human Kinetics Europe Ltd.

33. Stodden, D. F., Gao, Z., Goodway, J. D., \& Langendorfer, S. J. (2014). Dynamic relationships between motor skill competence and health-related fitness in youth. $\mathrm{Pe}$ diatric Exercise Science, 26(3), 231-241

34. Stodden, D.F, Goodway, J.D., Lagendorfer, S.J., Robertson, M.A., Rudisill, M.E., Garcia, C., \& Garcia, L.E. (2008). A Developmental Perspective on the Role of Motor Skill Competence in Physical Activity: An Emergent Relationship. Quest, 60, 290306

35. Westendorp, M., Houwen, S., Hartman, E., \& Visscher, C. (2011). Are gross motor skills and sports participation related in children with intellectual disabilities?. Research in Developmental Disabilities, 32(3), 1147-1153

36. Whitehead, M. (2019). Physical literacy across the world. Routledge

37. Whitehead, M. (2001). The Concept of Physical Literacy. European Journal of Physical Education, 6(2), 127-38

38. Whitehead, M. (2013). Definition of Physical Literacy and Clarification of Related Issues. ICSSPE Bulletin, 65, 29-34

39. Whitehead, M. (Ed.). (2010). Physical literacy: Throughout the Lifecourse. Routledge.

40. Zask, A., Barnett, L. M., Rose, L., Brooks, L. O., Molyneux, M., Hughes, D., Adams, J., \& Salmon, J. (2012). Three year follow-up of an early childhood intervention: is movement skill sustained?. The International Journal of Behavioral Nutrition and Physical Activity, 9, 127 